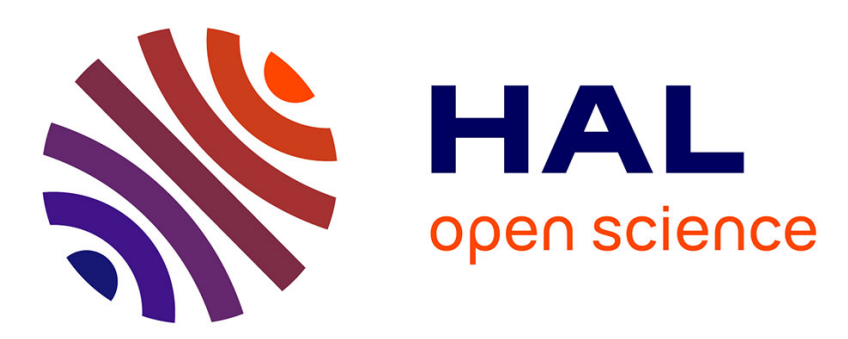

\title{
Analytical investigation of periodic solutions for a coupled oscillator with dry friction \\ Madeleine Pascal
}

\section{To cite this version:}

Madeleine Pascal. Analytical investigation of periodic solutions for a coupled oscillator with dry friction. ASME International Design Engineering Technical Conferences and Computers and Information in Engineering Conference 2009 (DETC2009), Aug 2009, San Diego, CA, United States. pp.399-406, 10.1115/DETC2009-86087 . hal-00747525

\section{HAL Id: hal-00747525 \\ https://hal.science/hal-00747525}

Submitted on 10 Jun 2016

HAL is a multi-disciplinary open access archive for the deposit and dissemination of scientific research documents, whether they are published or not. The documents may come from teaching and research institutions in France or abroad, or from public or private research centers.
L'archive ouverte pluridisciplinaire HAL, est destinée au dépôt et à la diffusion de documents scientifiques de niveau recherche, publiés ou non, émanant des établissements d'enseignement et de recherche français ou étrangers, des laboratoires publics ou privés. 


\title{
Analytical investigation of periodic solutions for a coupled oscillator with dry friction
}

\author{
Madeleine PASCAL \\ Universite d'Evry Val d'Essonne, \\ Laboratoire IBISC, 40 rue du Pelvoux, \\ 92330, Evry, France \\ 33169477521,mpascal@iup.univ-evry.fr
}

\begin{abstract}
In this paper, we present an analytical method to investigate the behavior of a two degrees of freedom oscillator excited by dry friction. The system consists of two masses connected by linear springs. These two masses are in contact with a driving belt moving at a constant velocity. The contact forces between the masses and the belt are obtained from Coulomb's friction laws. A set of periodic solutions involving a global sticking phase followed by several other phases where one or both masses are slipping, are found in close form. Stability conditions related to these solutions are obtained.
\end{abstract}

\section{INTRODUCTION}

Vibrating systems excited by dry friction are frequently encountered in technical applications. These systems are strongly non-linear and they are usually modeled as spring-mass oscillators. This paper is the continuation of the work [1], where we present an analytical method to investigate the behavior of a two degrees of freedom oscillator with dry friction. In this former paper, two kinds of periodic solutions including stick slip phases were found. In the following, a new kind of periodic motion is investigated, involving for each period a global stick phase [2] for each masses, followed by several other phases where one or both masses are slipping.

\section{NOMENCLATURE}

$\begin{array}{ll}\mathrm{C}, \mathrm{D}, \mathrm{H}, \Gamma & =4 \text { by } 4 \text { matrices depending on time. } \\ F_{1}, F_{2} & =\text { dry friction forces. } \\ J, J_{a}, J_{b}, J_{c}, J_{f} & =\text { Jacobian matrices. } \\ K, K_{1}, K_{2} & =\text { stiffness matrices. } \\ \mathrm{V} & =\text { belt velocity. } \\ k_{1}, k_{2} & =\text { springs stiffness. }\end{array}$

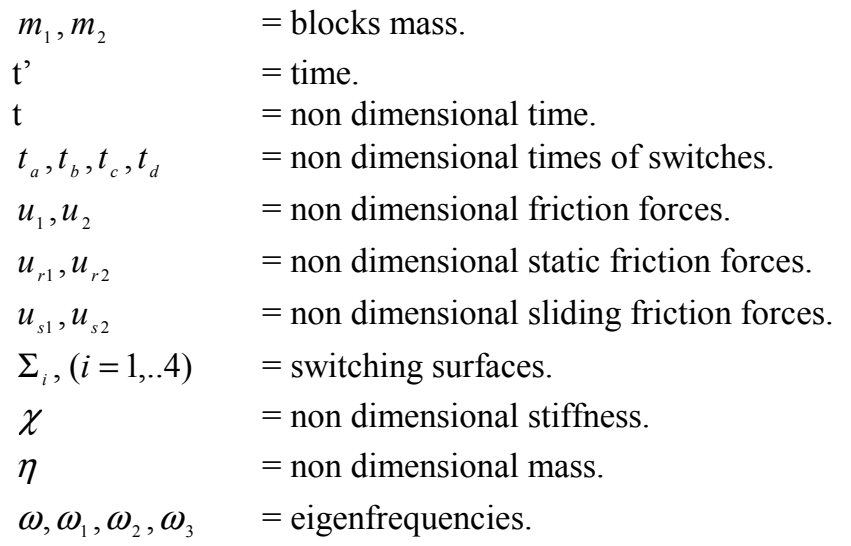

DESCRIPTION OF THE MODEL

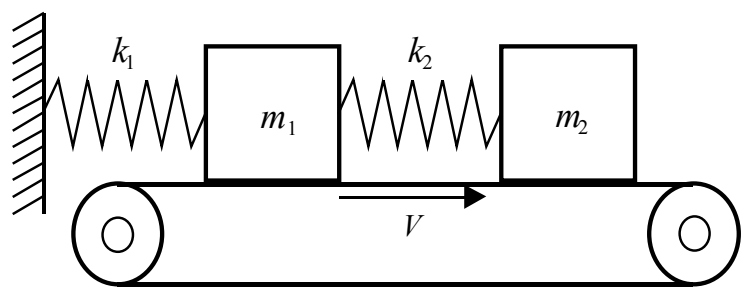

Figure 1: Coupled oscillator with dry friction.

The system consists of two masses $m_{1}, m_{2}$ connected by linear springs of stiffness $k_{1}, k_{2}$ (Fig.1). These two masses are in contact with a driving belt moving at a constant velocity. The contact forces $F_{1}, F_{2}$ between the masses and the belt are obtained assuming Coulomb's friction laws. 
The motion equations are written in the following form

$x_{1}^{\prime \prime}+x_{1}-\chi x_{2}=u_{1}, \quad \chi=\frac{k_{2}}{k_{1}+k_{2}}, \quad \eta=\frac{m_{1}}{m_{2}}$

$x_{2}^{\prime \prime}+\chi \eta\left(x_{2}-x_{1}\right)=\eta u_{2}, \quad u_{i}=\frac{F_{i}}{k_{1}+k_{2}}(i=1,2)$

$t=\omega_{3} t^{\prime}, \omega_{3}=\sqrt{\frac{k_{1}+k_{2}}{m_{1}}},\left(o^{\prime}\right)=\frac{d(o)}{d t}$

The friction forces are obtained from Coulomb's laws

- $V-x_{i}^{\prime} \neq 0, u_{i}=u_{s i} \operatorname{sign}\left(V-x_{i}^{\prime}\right)(i=1,2)\left(\operatorname{slip}\right.$ motion of $\left.m_{1}, m_{2}\right)$

- $V-x_{1}^{\prime}=0$

$u_{1}=\left\{\begin{array}{l}x_{1}-\chi x_{2} \text { if }\left|x_{1}-\chi x_{2}\right|<u_{r 1} \quad\left(\text { stick motion of } m_{1}\right) \\ \varepsilon u_{s 1} \text { if } \varepsilon\left(x_{1}-\chi x_{2}\right)>u_{r 1}(\varepsilon= \pm 1) \quad\left(\text { slip motion of } m_{1}\right)\end{array}\right.$

- $V-x_{2}^{\prime}=0$

$u_{2}=\left\{\begin{array}{l}\chi\left(x_{2}-x_{1}\right) \text { if }\left|\chi\left(x_{2}-x_{1}\right)\right|<u_{r 2}\left(\text { stick motion of } m_{2}\right) \\ \left.\varepsilon u_{s 2} \text { if } \varepsilon \chi\left(x_{2}-x_{1}\right)>u_{r 2}(\varepsilon= \pm 1) \text { (slip motion of } m_{2}\right)\end{array}\right.$

$u_{s i}, u_{s i},\left(u_{s i}<u_{r i}\right)(i=1,2)$ are the sliding and the sticking friction forces.

\section{PREDICTION OF THE OSCILLATIONS EXHIBITED BY \\ THE SYSTEM.}

The dynamical behavior of this piecewise linear system is very complex and includes slip or stick oscillations of the masses. All possible motions of the system are composed of several phases of slip motion for the both masses, stick motion of $m_{1}$ and slip motion of $m_{2}$ or vice versa and at last, stick motion for the two masses.

For each kind of motions, the close form solution is obtained. Global slipping phase: The equations of motion in this case are written as :

$$
\begin{aligned}
& z^{\prime \prime}+K z=0, \quad K=\left(\begin{array}{cc}
1 & -\chi \\
-\chi \eta & \chi \eta
\end{array}\right) \quad z=\left(z_{1}, z_{2}\right)^{t}, z_{i}=x_{i}-d_{0 i} \\
& z^{\prime}{ }_{i}<V,(i=1,2), d_{01}=\frac{u_{s 1}+u_{s 2}}{1-\chi}, d_{02}=\frac{u_{s 1} \chi+u_{s 2}}{(1-\chi) \chi}
\end{aligned}
$$

The solution is given by

$$
Z(t)=H(t) Z_{0}, \quad Z=\left(\begin{array}{l}
z \\
z^{\prime}
\end{array}\right), Z_{0}=Z(0)
$$

The 4 by 4 matrix $H(t)$ is obtained in analytical form [1], by a modal analysis of the system (3) (see also Appendix A) Slip-stick motion:(slip motion of $m_{1}$, stick motion of $m_{2}$ )

$$
\begin{aligned}
& z^{\prime \prime}+K_{1} z=0, \quad K_{1}=\left(\begin{array}{rr}
1 & -\chi \\
0 & 0
\end{array}\right) \\
& z_{1}^{\prime}<V, z_{2}^{\prime}=V, e_{2}-2 u_{r 2}<\chi\left(z_{2}-z_{1}\right)<e_{2}, \quad\left(e_{2}=u_{r 2}-u_{s 2}\right)
\end{aligned}
$$

The solution is obtained from a modal analysis of (5), [1].

$Z(t)=\Gamma(t) Z_{0}$

The 4 by 4 matrix $\Gamma(t)$ is given in analytical form (Appendix A) Stick-slip motion:(stick motion of $m_{1}$, slip motion of $m_{2}$ )

$$
\begin{aligned}
& z^{\prime \prime}+K_{2} z=0, \quad K_{2}=\left(\begin{array}{ll}
0 & 0 \\
-\chi \eta & \chi \eta
\end{array}\right) \\
& z_{1}^{\prime}=V, z_{2}^{\prime}<V, e_{1}-2 u_{r 1}<z_{1}-\chi z_{2}<e_{1}, \\
& \left(e_{1}=u_{r 1}-u_{s 1}\right)
\end{aligned}
$$

The solution [1] is given by

$$
Z(t)=C(t) Z_{0}
$$

The 4 by 4 matrix $C(t)$ (Appendix A) is obtained by a modal analysis of (7).

Global sticking phase

$$
\begin{aligned}
& z^{\prime \prime}=0, z^{\prime}{ }_{1}=z^{\prime}{ }_{2}=V, \quad e_{1}-2 u_{r 1}<z_{10}-\chi z_{20}<e_{1} \\
& e_{2}-2 u_{r 2}<\chi\left(z_{20}-z_{10}\right)<e_{2}
\end{aligned}
$$

The solution in this case is written as

$Z(t)=D(t) Z_{0}, \quad D=\left(\begin{array}{cc}I & V t I \\ 0 & I\end{array}\right), \quad I=\left(\begin{array}{ll}1 & 0 \\ 0 & 1\end{array}\right)$

\section{PERIODIC SOLUTIONS INCLUDING A GLOBAL STICK PHASE.}

In contrast with the periodic solutions investigated in [1], a new kind of periodic orbits is found, which includes a sticking phase for both masses, followed by three other phases where one or two masses are slipping. For each parts of the motion, the solution is obtained in analytical form. The instants of the transitions between one part of the motion to another one are obtained by writing the switching conditions.

A transition from slip to stick motion occurs if at some instant, the velocity of the mass reaches the velocity of the belt and if at this time, the restoring force applied to the mass is smaller than the corresponding static friction force.

A transition from stick to slip motion occurs if at some instant, the restoring force applied to the mass reaches the value of the static friction force.

Let us assume that at $\mathrm{t}=0$ 


$$
\begin{aligned}
& z_{i 0}^{\prime}=V,(i=1,2), \quad e_{1}-2 u_{r 1}<z_{10}-\chi z_{20}<e_{1}, \\
& e_{2}-2 u_{r 2}<\chi\left(z_{20}-z_{10}\right)<e_{2}
\end{aligned}
$$

For $0<t<t_{a}$, the two masses perform a stick motion given by (10). This motion ends when one of the restoring forces applied to the masses reaches the limiting value $u_{r i}$. For all the motion, the restoring force applied to the second mass is constant $\left(z_{2}-z_{1}=z_{20}-z_{10}\right)$. A transition occurs at $t=t_{a}$, when the restoring force applied to the first mass reaches its limiting value. Let us define:

$$
\begin{aligned}
& Z_{a} \equiv Z\left(t_{a}\right)=D\left(t_{a}\right) Z_{0}, \quad Z_{a}=\left(\begin{array}{c}
z_{a} \\
z^{\prime}{ }_{a}
\end{array}\right), z_{a}^{\prime}=(V, V)^{t}, \\
& z_{a}=\left(z_{1 a}, z_{2 a}\right)^{t}, \quad z_{1 a}-\chi z_{2 a}=e_{1}
\end{aligned}
$$

For a new period of time : $t_{a}<t<t_{a}+t_{b}, M_{1}$ performs a slip motion, while $M_{2}$ is still in stick motion:

$$
Z(t)=\Gamma\left(t-t_{a}\right) Z_{a}
$$

This motion ends at $t=t_{a}+t_{b}$, when the restoring force applied to the second mass reaches its limiting value. Let us define

$Z_{b} \equiv Z\left(t_{a}+t_{b}\right)=\Gamma\left(t_{b}\right) Z_{a}, \quad Z_{b}=\left(\begin{array}{c}z_{b} \\ z_{b}^{\prime}\end{array}\right)$,

$z_{b}^{\prime}=\left(z_{1 b}^{\prime}, V\right)^{t}, z_{b}=\left(z_{1 b}, z_{2 b}\right)^{t}, \quad \chi\left(z_{2 b}-z_{1 b}\right)=e_{2}$

A new phase occurs for $t_{a}+t_{b}<t<t_{a}+t_{b}+t_{c}$, related to a global slip motion of the system, defined by

$$
Z(t)=H\left(t-t_{a}-t_{b}\right) Z_{b}
$$

This motion ends at $t=t_{a}+t_{b}+t_{c}$, if at that time

$$
\begin{aligned}
z_{1 c}^{\prime} & \equiv z_{1}^{\prime}\left(t_{a}+t_{b}+t_{c}\right)=V, e_{1}-2 u_{r 1}<z_{1 c}-\chi z_{2 c}<e_{1}, \\
z_{i c} & =z_{i}\left(t_{a}+t_{b}+t_{c}\right),(i=1,2)
\end{aligned}
$$

For $t_{a}+t_{b}+t_{c}<t<t_{a}+t_{b}+t_{c}+t_{d}$, the first mass performs a stick motion $\left(z^{\prime}{ }_{1}=V\right)$, while the second one performs a slip motion

$$
Z(t)=C\left(t-t_{a}-t_{b}-t_{c}\right) Z_{c}, Z_{c}=Z\left(t_{a}+t_{b}+t_{c}\right)
$$

A periodic motion of period $t_{a}+t_{b}+t_{c}+t_{d}$ is obtained if

$$
Z\left(t_{a}+t_{b}+t_{c}+t_{d}\right)=Z_{0}
$$

The obtained periodic motion depends of 6 parameters: $z_{10}, z_{20}, t_{a}, t_{b}, t_{c}, t_{d}$ and the switching conditions (12), (14), (16), together with the periodicity conditions (18) provide 6 scalar equations for the determination of these parameters. This new periodic motion is a generalization of the solution II obtained in [1]: this last solution is a particular case of this new solution for $t_{a}=0$.

\section{STABILITY OF THE PERIODIC SOLUTION.}

The dry friction oscillator considered in this paper is not smooth, with a phase space dimension varying between two (global stick motion) and four ( global slip motion). Due to the presence of discontinuities in the equations of motion, the stability of the periodic solutions cannot be investigated by the classical linearization method An alternative way [3] consists to use Poincarre maps modeling. The phase space is partitioned into four configurations (Fig.2).

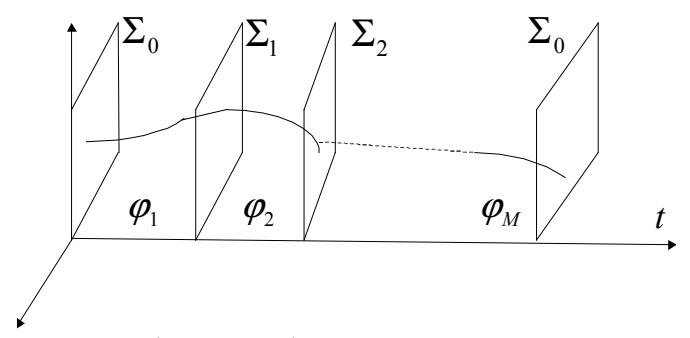

Figure 2: Phase space

At the boundaries $\left(\Sigma_{0}, \Sigma_{1}, . . \Sigma_{4} \equiv \Sigma_{0}\right)$ between two adjacent configurations, the system switches from one configuration to another. Within each configuration, the close form solution is known

$$
\begin{aligned}
& Z(t)=\varphi_{k}\left(t-t_{k-1}\right) Z\left(t_{k-1}\right),(k=1, \ldots, 4), \\
& \left(\varphi_{1}=D, \varphi_{2}=\Gamma, \varphi_{3}=H, \varphi_{4}=C\right)
\end{aligned}
$$

The switching moments

$t_{i}(i=1, . ., 4)=\left(t_{a}, t_{a}+t_{b}, t_{a}+t_{b}+t_{c}, t_{a}+t_{b}+t_{c}+t_{d}\right)$

are obtained as solution of the switching conditions. A Poincarre map from $\left(\Sigma_{0}\right)$ to $\left(\Sigma_{0}\right)$ is defined as $P=P_{4} \circ . . \circ P_{1}$, where $P_{k}$ is a local mapping between $\left(\Sigma_{k-1}\right)$ and $\left(\Sigma_{k}\right)$ defined by

$$
P_{k}: Z\left(t_{k-1}\right) \rightarrow Z\left(t_{k}\right)=\varphi_{k}\left(t_{k}-t_{k-1}\right) Z\left(t_{k-1}\right), \quad(k=1, . ., 4)
$$

The obtained periodic solutions are related to the fixed points of this map. The stability of these fixed points depends on the eigenvalues of the Jacobian matrix $J$ of the map.

If one eigenvalue of $J$ (at least) is outside the unit circle, the fixed point of the map is unstable and the corresponding periodic solution is also unstable. If all the eigenvalues lie inside the unit circle, the fixed point of the map is stable, but the corresponding periodic orbit is only conditionally stable, with 
respect to some particular perturbations of the initial conditions leading to the same kind of switches.

Let us introduce small perturbations into the initial conditions $z_{00}, z^{\prime}{ }_{00}$ leading to a periodic solution

$z_{0}=z_{00}+d z_{0}, z_{0}^{\prime}=z_{00}^{\prime}+d z^{\prime}, d z_{0}=(x, y)^{t}, d z_{0}^{\prime}=(u, v)^{t}$

Moreover, the new initial conditions are assumed to lie in the map of the phase space defined by

$z_{1}^{\prime}=z_{2}^{\prime}=V$, i.e. $u=v=0$

If the initial perturbations $x, y$ are small, the restoring forces applied to the masses are smaller than their limiting values, and the perturbed motion is a global stick motion given by:

$Z(t)=D(t) Z_{0}, Z_{0}=\left(\begin{array}{c}z_{0} \\ z^{\prime}{ }_{0}\end{array}\right)$

This motion ends at $t=t_{a}+d t_{a}$, when the restoring force applied to the first mass reaches the limiting value $u_{r 1}$.

$Z\left(t_{a}+d t_{a}\right)=D\left(t_{a}+d t_{a}\right)\left(Z_{00}+d Z_{0}\right)$

Assuming small perturbations

$d Z_{a}=Z\left(t_{a}+d t_{a}\right)-Z\left(t_{a}\right)=D\left(t_{a}\right) d Z_{0}+D^{\prime}\left(t_{a}\right) Z_{00} d t_{a}$

$d Z_{a}=\left(\begin{array}{l}d z_{a} \\ d z^{\prime}{ }_{a}\end{array}\right), d z_{a}=\left(\begin{array}{c}x_{a} \\ y_{a}\end{array}\right), d z^{\prime}{ }_{a}=0$

The value of $d t_{a}$ is obtained from the condition

$x_{1}\left(t_{a}+d t_{a}\right)-\chi x_{2}\left(t_{a}+d t_{a}\right)=u_{r 1}$, i.e. $x_{a}=\chi y_{a}$

From (24), (25), we deduce

$y_{a}=J_{a}\left(\begin{array}{l}x \\ y\end{array}\right), J_{a}=\frac{1}{1-\chi}(-1,1)$

The next step is a slip stick motion given by:

$Z(t)=\Gamma\left(t-t_{a}-d t_{a}\right)\left(Z_{a}+d Z_{a}\right)$

This motion ends at $t=t_{a}+d t_{a}+t_{b}+d t_{b}$, when the restoring force applied to the second mass reaches the limiting value $u_{r 2}$.

$Z\left(t_{a}+d t_{a}+t_{b}+d t_{b}\right)=\Gamma\left(t_{b}+d t_{b}\right)\left(Z_{b}+d Z_{b}\right)$

Assuming small perturbations $d Z_{b}=Z\left(t_{a}+d t_{a}+t_{b}+d t_{b}\right)-Z\left(t_{a}+t_{b}\right)$

$=\Gamma\left(t_{b}\right) d Z_{b}+\Gamma^{\prime}\left(t_{b}\right) Z_{b} d t_{b}$

$d Z_{b}=\left(\begin{array}{l}d z_{b} \\ d z^{\prime}{ }_{b}\end{array}\right), d z_{b}=\left(\begin{array}{l}x_{b} \\ y_{b}\end{array}\right), d z^{\prime}{ }_{b}=\left(\begin{array}{l}u_{b} \\ 0\end{array}\right)$

The value of $d t_{b}$ is obtained from the condition

$\chi\left(x_{2}\left(t_{a}+d t_{a}+t_{b}+d t_{b}\right)-x_{1}\left(t_{a}+d t_{a}+t_{b}+d t_{b}\right)\right)=u_{r 1}$, i.e. $x_{b}=y_{b}$

We deduce

$$
\begin{aligned}
& \left(\begin{array}{l}
y_{b} \\
u_{b}
\end{array}\right)=J_{b} y_{b}, J_{b}=\left(\begin{array}{l}
\tilde{\gamma}_{1} \\
\tilde{\gamma}_{2}
\end{array}\right), \tilde{\gamma}_{1}=\frac{\chi-\chi_{b}}{1-\chi_{b}}, \\
& \tilde{\gamma}_{2}=\left(\chi z_{2 b}-z_{1 b}\right) \frac{(\chi-1)}{V\left(1-\chi_{b}\right)} \\
& \chi_{b}=z^{\prime}{ }_{1}\left(t_{b}\right) / V,(i=1,2)
\end{aligned}
$$

A new step is a global slip motion given by

$Z(t)=H\left(t-t_{a}-d t_{a}-t_{b}-d t_{b}\right)\left(Z_{b}+d Z_{b}\right)$

A transition occurs at $t=t_{a}+d t_{a}+t_{b}+d t_{b}+t_{c}+d t_{c}$, when the velocity of the first mass reaches the velocity $\mathrm{V}$ of the belt, the restoring force applied to this mass being less than its limiting value for small perturbations.

$d Z_{c}=Z\left(t_{a}+d t_{a}+t_{b}+d t_{b}+t_{c}+d t_{c}\right)-Z\left(t_{a}+t_{b}+t_{c}\right)$

$=H\left(t_{c}\right) d Z_{b}+H^{\prime}\left(t_{c}\right) Z_{b} d t_{c}$

$d Z_{c}=\left(\begin{array}{l}d z_{c} \\ d z^{\prime}{ }_{c}\end{array}\right), d z_{c}=\left(\begin{array}{l}x_{c} \\ y_{c}\end{array}\right), d z^{\prime}{ }_{c}=\left(\begin{array}{l}u_{c} \\ v_{c}\end{array}\right)$

The value of $d t$ is obtained from the condition

$x_{1}^{\prime}\left(t_{a}+d t_{a}+t_{b}+d t_{b}+t_{c}+d t_{c}\right)=V$,
i.e. $u_{c}=0$

It results

$\left(\begin{array}{l}x_{c} \\ y_{c} \\ v_{c}\end{array}\right)=J_{c}\left(\begin{array}{l}x_{b} \\ u_{b}\end{array}\right)$ 
$J_{c}=\left(\begin{array}{l}\left(H_{11}+H_{12}+\tilde{k}_{1}\left(H_{31}+H_{32}\right), H_{13}+\widetilde{k}_{1} H_{11}\right. \\ \left(H_{21}+H_{22}+\widetilde{k}_{2}\left(H_{31}+H_{32}\right), H_{23}+\widetilde{k}_{2} H_{11}\right. \\ \left(H_{41}+H_{42}+\widetilde{k}_{3}\left(H_{31}+H_{32}\right), H_{21}+\widetilde{k}_{3} H_{11}\right.\end{array}\right)$

$H_{i j}=H_{i j}\left(t_{c}\right),(i, j=1,2,3), \tilde{k}_{1}=\frac{V}{z_{1 c}-\chi z_{2 c}}$,

$\widetilde{k}_{2}=\widetilde{k}_{1} z_{2 c}^{\prime} / V, \widetilde{k}_{3}=\widetilde{k}_{1} \chi \eta\left(z_{1 c}-z_{2 c}\right) / V$

The last step is a stick slip motion given by

$Z(t)=C\left(t-t_{a}-d t_{a}-t_{b}-d t_{b}-t_{c}-d t_{c}\right)\left(Z_{c}+d Z_{c}\right)$

This motion finishes at

$t=t_{a}+d t_{a}+t_{b}+d t_{b}+t_{c}+d t_{c}+t_{d}+d t_{d}$, when the

velocity of the second mass reaches the velocity of the belt.

$d Z_{f}=Z\left(t_{a}+d t_{a}+t_{b}+d t_{b}+t_{c}+d t_{c}+t_{d}+d t_{d}\right)$

$-Z\left(t_{a}+t_{b}+t_{c}+t_{d}\right)=C\left(t_{d}\right) d Z_{c}+C^{\prime}\left(t_{d}\right) Z_{c} d t_{d}$

$d Z_{f}=\left(\begin{array}{l}d z_{f} \\ d z_{f}^{\prime}\end{array}\right), d z_{f}=\left(\begin{array}{l}x_{f} \\ y_{f}\end{array}\right), d z_{f}^{\prime}=\left(\begin{array}{l}0 \\ v_{f}\end{array}\right)$

The value of $d t_{d}$ is obtained from the condition

$x_{2}^{\prime}\left(t_{a}+d t_{a}+t_{b}+d t_{b}+t_{c}+d t_{c}+t_{d}+d t_{d}\right)=V$,

i.e. $v_{f}=0$

We deduce

$\left(\begin{array}{l}x_{f} \\ y_{f}\end{array}\right)=J_{f}\left(\begin{array}{l}x_{c} \\ y_{c} \\ v_{c}\end{array}\right), J_{f}=\left(\begin{array}{cc}1+\tilde{k}_{4}, & -\tilde{k}_{4}, \tilde{k}_{5} \\ 1-\tilde{k}_{6}, & \tilde{k}_{6}, \tilde{k}_{7}\end{array}\right)$

$\tilde{k}_{4}=\frac{\sin \left(\omega t_{d}\right)}{V \omega\left(z_{200}-z_{100}\right)}, \tilde{k}_{5}=\frac{\tilde{k}_{4}}{\omega} \cot \left(\omega t_{d}\right)$,

$\tilde{k}_{6}=\cos \left(\omega t_{d}\right)-\tilde{k}_{4}, \tilde{k}_{7}=\frac{\sin \left(\omega t_{d}\right)}{\omega}+\tilde{k}_{5}$,

$\left(\omega^{2}=\chi \eta\right)$

The linear correspondence between the initial and the final perturbations is then obtained

$\left(\begin{array}{l}x_{f} \\ y_{f}\end{array}\right)=J\left(\begin{array}{l}x \\ y\end{array}\right), J=J_{f} J_{c} J_{b} J_{a}$

The stability of the periodic solution depends on the eigenvalues of the matrix $J$. If all these eigenvalues lie inside the unit circle, the periodic solution is conditionally stable. If one of them at least is outside this circle, the periodic solution is unstable.

The eigenvalues of the $J$ matrix are the roots of the equation

$P(\lambda) \equiv \operatorname{det}(J-\lambda I)=0$

$J$ is a singular matrix

$\operatorname{det}(J)=\operatorname{det}\left(J_{f} J_{c}\right) \operatorname{det}\left(J_{b} J_{a}\right)$,

$\operatorname{det}\left(J_{b} J_{a}\right)=0$

It results that: $P(\lambda)=\lambda(\lambda-\operatorname{tr}(J))$

The periodic solution is conditionally stable if $|\operatorname{tr}(J)<1|$.

Otherwise, the periodic solution is unstable.

\section{NUMERICAL VALIDATION}

The periodicity conditions (18) gives the following equations

$\left(N_{1}-I\right) z_{0}+N_{2} z_{0}^{\prime}=0$

$\begin{aligned} & N_{3} z_{0}+\left(N_{4}-I\right) z_{0}^{\prime}=0 \\ & N=C\left(t_{d}\right) H\left(t_{c}\right) \Gamma\left(t_{b}\right) D\left(t_{a}\right)\end{aligned} \quad N=\left(\begin{array}{ll}N_{1} & N_{2} \\ N_{2} & N_{4}\end{array}\right)$

$z^{\prime}{ }_{0}=(V, V)^{t}$

These periodicity conditions include the last switching condition (16).

From (44), we deduce two compatibility conditions

$$
\begin{aligned}
& M_{11}+M_{12}=0 \\
& M_{21}+M_{22}=0 \\
& M=\left(N_{1}-I\right)^{-1} N_{2}-\left(N_{3}\right)^{-1}\left(N_{4}-I\right)
\end{aligned}
$$

The relation (45) provides two equations linking the time duration $\left(t_{a}, t_{b}, t_{c}, t_{d}\right)$ of each part of the motion; from (44), we deduce the value of $z_{0}$

$z_{0}=-\left(N_{1}-I\right)^{-1} N_{2} z_{0}^{\prime}$

Inserting this result in the switching condition (12) and (14), we obtain two new relations for the determination of the parameters $t_{a}, t_{b}, t_{c}, t_{d}$. Several conditions about the results obtained by this numerical method must be fulfilled: for example, the computed values of the time duration $t_{a}, t_{b}, t_{c}, t_{d}$ must be positive numbers.

For the following values of the data: $\chi=0.8, \eta=V=1, e_{1}=2.7844, e_{2}=0.8587$,

we obtain

$t_{a}=0.6784, t_{b}=2, t_{c}=1.5, t_{d}=2.34$,

$z_{10}=0.8921, z_{20}=-2.1958$ 
The corresponding behavior of the system is shown on Figures 3,4 . Each parts of the system's motion during one period are shown on these figures ( curves O1a1, O2a2: global sticking motion; curves a1b1,a2b2: slip stick motion; curvesb1c1,b2c2: global slipping motion; curves c1d1,c2d2: stick slip motion) .

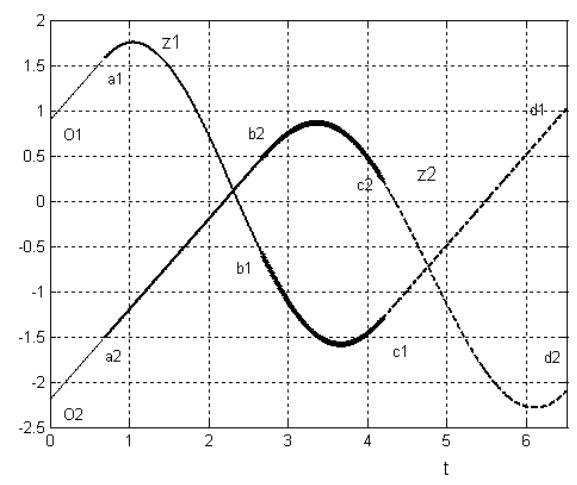

Figure 3: Time history of the masses.

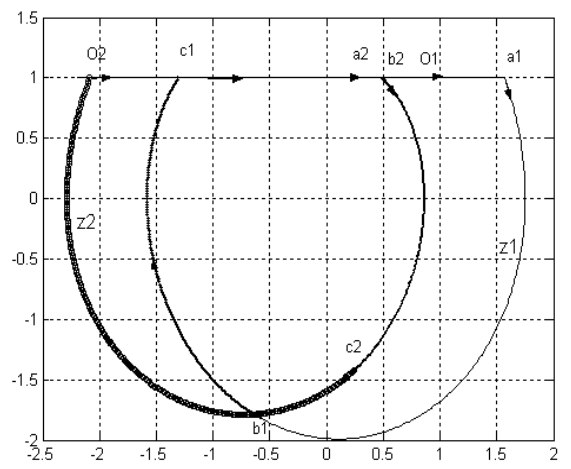

Figure 4: Phase plots of the system.

\section{CONCLUSION}

The system considered in this work is a non smooth system in presence of discontinuities of forces and motions. The corresponding dynamical model is nonlinear.

However, the system can be partitioned into different configurations. Within each configuration, the dynamical model is linear and the analytical solution of the motion equations is available. It results that, even in the case of multi degrees of freedom systems, it is possible to obtain the solution of these non-linear systems by patching the solutions of the successive configurations. Due to the presence of discontinuities, the stability of the periodic solutions cannot be investigated by the classical methods used for smooth systems. An alternative way consists to use a Poincarre map modeling. However, this method gives only some results about the conditional stability of the solution.

\section{REFERENCES}

[1] Pascal, M.,2008, "Dynamics of coupled oscillators excited by dry friction", J. of Computational and Nonlinear Dynamics ,Vol.3 (3), pp.1-6.

[2] Galvanetto, U., and Knudsen, C.,1997, "Event Maps in a Stick-Slip System", Nonlinear Dynamics, Vol. 13,pp. 99-115.

[3] Natsiavas, S.,1998, "Stability of piecewise linear oscillators with viscous and dry friction damping", J. Sound and Vibration , Vol.217 (3), pp. 507-522.

\section{APPENDIX A: MATRICES $H, \Gamma, C$.}

$$
\begin{aligned}
H & =\left(\begin{array}{ll}
H_{1} & H_{2} \\
H_{3} & H_{1}
\end{array}\right) \quad H_{i}=\Lambda B_{i} \Lambda^{-1} \quad(i=1,2,3) \\
\Lambda & =\left(\begin{array}{ll}
1 & 1 \\
\lambda_{1} & \lambda_{2}
\end{array}\right) B_{1}=\left(\begin{array}{cc}
c_{1} & 0 \\
0 & c_{2}
\end{array}\right), \\
B_{2} & =\left(\begin{array}{ll}
\frac{s_{1}}{\omega_{1}} & 0 \\
0 & \frac{s_{2}}{\omega_{2}}
\end{array}\right), \quad B_{3}=B_{1}^{\prime} \\
c_{i} & =\cos \left(\omega_{i} t\right), \quad s_{i}=\sin \left(\omega_{i} t\right), \quad(i=1,2)
\end{aligned}
$$

$\omega_{1}, \omega_{2}$ are the roots of the characteristic equation

$\operatorname{det}\left(K-I \omega^{2}\right)=0, \psi_{i}=\left(\begin{array}{l}1 \\ \lambda_{i}\end{array}\right),(i=1,2)$

$\left(K-I \omega_{i}^{2}\right) \psi_{i}=0$.

$\Gamma=\left(\begin{array}{ll}\Gamma_{1} & \Gamma_{2} \\ \Gamma_{3} & \Gamma_{1}\end{array}\right), \quad \Gamma_{i}=\Sigma \gamma_{i} \Sigma^{-1},(i=1,2,3)$

$\Sigma=\left(\begin{array}{ll}1 & \chi \\ 0 & 1\end{array}\right), \gamma_{1}=\left(\begin{array}{cc}\cos t & 0 \\ 0 & 1\end{array}\right)$,

$\gamma_{2}=\left(\begin{array}{ll}\sin t & 0 \\ 0 & t\end{array}\right), \quad \gamma_{3}=\gamma_{1}^{\prime}$

$C=\left(\begin{array}{ll}C_{1} & C_{2} \\ C_{3} & C_{1}\end{array}\right)$ 
$C_{i}=\Pi A_{i} \Pi^{-1}(i=1,2,3), \Pi=\left(\begin{array}{ll}1 & 0 \\ 1 & 1\end{array}\right)$

$A_{1}=\left(\begin{array}{cc}1 & 0 \\ 0 & \cos (\omega t)\end{array}\right), A_{2}=\left(\begin{array}{cc}t & 0 \\ 0 & \frac{\sin (\omega t)}{\omega}\end{array}\right)$,

$A_{3}=A_{1}^{\prime}, \quad \omega^{2}=\chi \eta$

\section{APPENDIX B: CONSTRAINTS}

Several constraints must be fulfilled all along each parts of the motion and the obtained parameters $\left(z_{10}, z_{20}, t_{a}, t_{b}, t_{c}, t_{d}\right)$ are related to a realistic solution if some constraints are true. First, we need to obtain

$$
\begin{aligned}
& t_{a}>0, t_{b}>0, t_{c}>0, t_{d}>0 \\
& e_{1}-2 u_{r 1}<z_{10}-\chi z_{20}<e_{1} \\
& e_{2}-2 u_{r 2}<\chi\left(z_{20}-z_{10}\right)<e_{2}
\end{aligned}
$$

The numerical values obtained for the parameters $\left(z_{10}, z_{20}, t_{a}, t_{b}, t_{c}, t_{d}\right)$ fulfill almost all these constraints, excepted the constraint including the value of $u_{r 1}, u_{r 2}$. The solution depending only on $e_{1}, e_{2}$, the constraints (51), (52) are verified if we choose $u_{r 1}, u_{r 2}$ in such way that

$u_{r 1}>\frac{V t_{a}(1-\chi)}{2}, \quad u_{r 2}>\frac{e_{2}-\chi\left(z_{20}-z_{10}\right)}{2}$.

For the numerical validation, we obtain

$u_{r 1}>0.0678, \quad u_{r 2}>1.6645$.

The first condition is always verified: $\quad\left(u_{r 1}>e_{1}=2.7844\right)$

The other constraints are the following:

For $0<t<t_{a}$

$e_{1}-2 u_{r 1}<z_{1}-\chi z_{2}<e_{1}, \quad e_{2}-2 u_{r 2}<\chi\left(z_{2}-z_{1}\right)<e_{2}$

From

$z_{1}-\chi z_{2}=z_{10}-\chi z_{20}+V t(1-\chi)=e_{1}+V\left(t-t_{a}\right)(1-\chi)$

$\chi\left(z_{2}-z_{1}\right)=\chi\left(z_{20}-z_{10}\right)$

we deduce

$z_{10}-\chi z_{20}<z_{1}-\chi z_{2}<e_{1}$
Taking into account (52), (53),(55) and (56), the constraints (54) are fulfilled.

For $t_{a}<t<t_{a}+t_{b}$

$z_{1}^{\prime}<V, \quad e_{2}-2 u_{r 2}<h(t) \equiv \chi\left(z_{2}-z_{1}\right)<e_{2}$

The first constraint is fulfilled (Fig.4). On the other hand

$h^{\prime}(t)=\chi\left(z^{\prime}{ }_{2}-z_{1}^{\prime}\right)=\chi\left(V-z_{1}^{\prime}\right)>0$

The function $h(t)$ is an increasing function on the interval

$t_{a}<t<t_{a}+t_{b}$

$e_{2}-2 u_{r 2}<h\left(t_{a}\right)<e_{2}, h\left(t_{a}+t_{b}\right)=e_{2}$

The constraint (57) is satisfied.

For $t_{a}+t_{b}<t<t_{a}+t_{b}+t_{c}$, the constraint

$z_{1}^{\prime}<V, z_{2}{ }_{2}<V$ is fulfilled ( Fig.4)

For $t_{a}+t_{b}+t_{c}<t<t_{a}+t_{b}+t_{c}+t_{d}$

$e_{1}-2 u_{r 1}<z_{1}-\chi z_{2}<e_{1}, \quad z_{2}^{\prime}<V$

The second condition is fulfilled (Fig.4). Let us consider

$\bar{h}(t)=z_{1}-\chi z_{2}$

$\bar{h}^{\prime}(t)=z_{1}^{\prime}-\chi z^{\prime}{ }_{2}=V-\chi z^{\prime}{ }_{2}>0$

The function $\bar{h}^{\prime}(t)$ is an increasing function of t on the interval $\left(t_{a}+t_{b}+t_{c}<t<t_{a}+t_{b}+t_{c}+t_{d}\right)$. From

$\bar{h}\left(t_{a}+t_{b}+t_{c}+t_{d}\right)=z_{10}-\chi z_{20}$

we conclude that the first condition (59) is fulfilled. 